\title{
Notas sobre la flora vascular de Galápagos
}

\author{
Katya Romoleroux \\ Escuela de Ciencias Biológicas de la Pontificia Universidad Católica del Ecuador. \\ kromoleroux@puce.edu.ec
}

\section{Charles Darwin y la flora de Galápagos}

La colección botánica realizada por Darwin en Galápagos en 1835, cuando visitó las Islas en su viaje con el Beagle, fue la colección más completa en su tiempo; estas colecciones fueron enviadas al herbario en Cambridge y representó el incentivo para escribir el primer tratado sobre las plantas de Galápagos, publicado por J. D. Hooker (1847), llamado: An Enumeration of the plants of the Galápagos Archipiélago; with descriptions of those which are new.

Posteriormente se han producido varias publicaciones sobre la flora de Galápagos, algunas aún citan las colecciones de Darwin, como es: Flora of the Galápagos Islands de Wiggins \& Porter (1971), libro considerado como el estudio más completo de las plantas vasculares de Galápagos; recientemente, Porter (2009) publicó el artículo: "Some new Darwin vascular plant specimens from the Beagle voyage", en el cual cita especies de Darwin en Galápagos.

\section{Origen y dispersión de la flora vascular de Galápagos}

Por su origen, se pueden reconocer básicamente dos elementos fitogeográficos: el nativo y el exótico. El primero podría haber llegado gracias a agentes naturales de dispersión: agua, viento, animales, y puede clasificarse en elemento endémico, es decir, que sólo existe en Galápagos, y en no-endémico, lo cual significa que se encuentra también en otros lugares. El segundo, con seguridad, llegó por actividades humanas, y está en relación directa con las plantas introducidas accidentalmente o intencionalmente para cultivos.

Estudios realizados para tratar de cuantificar las relaciones geográficas de la flora vascular nativa de Galápagos indican que está cercanamente relacionada con la flora del continente americano, en especial con la de Sudamérica (80\% elemento Americano, 20\% elemento Pantropical). En cuanto a la dispersión, las aves han representado el factor más importante en la dispersión de las angiospermas nativas $-60 \%$ aves, $32 \%$ viento y $8 \%$ agua - (Porter, 1983).

\section{Características de la flora de Galápagos}

La flora de estas islas posee las siguientes características:

1. Composición florística inarmónica: las plantas dominantes son aquellas que presentan dispersión eficiente a 
larga distancia. Ejemplos: Helechos, Asteraceae, Cyperaceae, Poaceae, Amaranthaceae. Semillas con alto grado de latencia.

2. Principalmente herbácea y arbustiva.

3. Plantas en su mayoría hermafroditas y reproductivamente autocompatibles: domina la autopolinización, solo $3 \%$ de la flora es dioica.

\section{Zonas de vegetación en Galápagos}

Se pueden distinguir algunas zonas de vegetación, las cuales varían de acuerdo a la altitud y presentan vegetación característica. En la isla Santa Cruz, se pueden aún reconocer seis zonas de vegetación, en otras islas no están todas las zonas representadas.

Las seis zonas de vegetación, que están ordenadas por altitud desde 0 m.s.n.m., la zona litoral, hasta 860 m.s.n.m., la zona de helechos, son las siguientes:

1. Zona litoral, parte inundada representada por manglares.
2. Zona seca, dominada principalmente por especies de la familia Cactaceae.

3. Zona de transición, vegetación transicional de zona seca y arbustiva.

4. Zona de Scalesia, dominada por el género arbóreo endémico Scalesia.

5. Zona de Miconia, representada principalmente por el género Miconia.

6. Zona de helechos, zona más alta con helechos terrestres y un helecho arbóreo.

\section{Flora nativa y endémica vascular de Galápagos}

Las plantas vasculares nativas y endémicas se originaron de ancestros que seguramente llegaron del continente, y en el caso de las endémicas evolucionaron en las islas hasta formar especies y géneros distintos y únicos.

Existen seis géneros endémicos de Angiospermas: Scalesia, Darwiniothamus, Macraea y Lecocarpus (Asteraceae); Brachycereus y Jasminocereus (Cactaceae). Además varias especies y taxa infraespecíficas de otras familias y géneros (Tabla 1).

\begin{tabular}{|lccc|}
\hline Plantas vasculares & Nativas & Endémicas & \% Endémicas \\
\hline Especies & $560(500)$ & 180 & $32(36)$ \\
\hline $\begin{array}{l}\text { Taxones } \\
\text { (especies más taxa } \\
\text { infraespecíficas) }\end{array}$ & $610(550)$ & 230 & $38(42)$ \\
\hline
\end{tabular}

Tabla 1. Número de plantas vasculares nativas y endémicas de Galápagos (Los números entre paréntesis representan la cantidad mínima, ya que en algunas especies es difícil definir su origen. Tye, 2000). 


\section{Plantas vasculares exóticas en Galápagos}

Durante los últimos 35 años, el número de especies introducidas ha aumentado aceleradamente, las cifras son muy preocupantes. Las especies introducidas más agresivas dominan grandes áreas donde las especies nativas están desapareciendo. Actualmente, hay más especies exóticas que nativas en Galápagos (Tabla 2).

\section{Conclusiones}

Aunque la conservación en Galápagos ha sido relativamente exitosa, los cientos de plantas ya introducidos son una amenaza permanente. Las especies nativas más amenazadas, de acuerdo a los criterios de UICN, habitan generalmente en las islas más pobladas.

Con la extinción de la flora nativa también desaparecerán las comunidades y zonas de vegetación. Hay muchos estudios aún por hacer sobre la flora de Galápagos, los cuales no se podrán culminar si no se toman medidas efectivas para proteger este hábitat único, que jugó un papel tan importante en la Teoría de la Evolución de Darwin.

\begin{tabular}{|c|c|l|}
\hline $\begin{array}{c}\text { Número de especies } \\
\text { introducidas }\end{array}$ & Año de publicación & Autores \\
\hline 77 & 1971 & Wiggins \& Porter \\
\hline 259 & 1987 & Lawesson et al. \\
\hline 438 & 1997 & Mauchamp \\
\hline 700 & 2006 & FCD. \\
\hline
\end{tabular}

Tabla 2. Número de especies introducidas en Galápagos, año y autores de la publicación.

\section{BIBLIOGRAFÍA}

FCD (Fundación Charles Darwin para las islas Galápagos) 2006. Plantas introducidas http://www.darwinfoundation.org/es/galapagos/species/Plantas /introduced

Hooker, J. D. 1847. An Enumeration of the plants of the Galápagos Archipiélago; with descriptions of those which are new. Trans. Linn. Soc. Bot. 20: 163-233.

Lawesson, J. E. Adersen, H. \& Bentley, P. 1987. An updated and annotated check list of the vascular plants of the Galapagos Islands. Reports from the Botanical Institute, University of Aarhus, n. ${ }^{\circ} 16.74 \mathrm{pp}$.

Mauchamp, A. 1997. Threats from alien plant species in the Galápagos Islandas. Conservation Biology 11260-263.
Porter, D. 1983. Vascular Plants of the Galápagos: origins and dispersal En: Bowman, R.I. Berson, M. \& Leviton, A.E. (Eds), Patterns of Evolution in Galapagos Organisms, Pacific Division, AAAS, San Francisco, CA, pp. 33-96.

Porter, D. 2009. Some new Darwin vascular plant specimens from the Beagle voyage. The Linnean Society of London, Botanical Journal of the Linnean Society, 2009, 159, 12-18.

Wiggins, I. \& Porter, D. 1971. Flora of the Galápagos Islands, Stanford University Press. California.

Tye, A. 2000. Plantas vasculares endémicas de Galápagos. En: Valencia R., Pitman, N. León-Yánez \& Jorgensen, P. (editores), Libro Rojo de las Plantas endémicas del Ecuador 2000. 